\title{
BASIC, ASPECTS OF SUPERALLOY DESIGN FOR CRACK GROWTH RESISTANCE
}

John K. Tien and Sampath Purushothaman

Henry Krumb School of Mines, Columbia University

New York, New York 10027

\begin{abstract}
In anticipation of the design of superalloy components by the fracture mechanics concept, we review and extend in this paper the current understanding of $f l a w$ or crack growth resistance. We develop theories for fatigue and creep crack growth resistance that are particularly applicable to supcralloys, including considerations of the crystallographic cracking which is unique to the nickel-base superalloys. For superalloys of a given strength level, it is predicted that fatigue crack growth resistance can be enhanced by increased plane strain ductility or cyclic work hardening exponent or elastic modulus. Based on our theories, and substantiated by very limited empirical data, it is believed that some nickel-base superalloys may not exhibit fail-safe fatigue crack growth behavior at low values of stress intensity. Cobalt-base superalloys may display this behavior, but superimposed brittle fracture modes could be present in these systems. Creep crack growth resistance is also discussed from alloy design viewpoints. Directions for future empirical research are indicated.
\end{abstract}




\section{INTRODUCTION}

It can now be bragged that rational and scientific approackes are available to successfully design superalloys in meeting certain specific needs. Understanding the role of chromium and aluminum in oxidation and hot corrosion resistance, in conjunction with basic knowledge of coherent precipitation and solid solution strengthening, can be applied to rationalize, for example, the evolution of the corrosion resistant $1 \mathrm{~N}-792$ from $1 \mathrm{~N}-100$, in-738 from $1 \mathrm{~N}-713$, and Ud-71.0 from Ud-700. Understanding the rules of stability and formation of alloy phases must have played a role in the invention of sigma-free Ud-700. Advances in casting techniques combined with the understanding of strong-directions and weak-links have given us the directional structures and practical single crystals. Appreciation for the mechanisms of precipitation strengthening and oxide dispersion strengthening, in conjunction with some elegant powder metallurgy, has resulted in combination alloys with advantages at highel temperatures, and hopefully, without unforeseen synergistic disadvantages. Certainly this appreciation for mixed marriages has opened a new avenue for superalloy design. Although the mechanisms are still resisting full understanding, the $B C$ and $H F$ concepts are now threatening to at least partially resolve that perennial conflict between strength and ductility. The examples go on.

What of the future? Should science or long range alloy research bow out gracefully from the superalloy scene and rest comfortably on its laurels? Perhaps this is self-serving, but we answer no because there is at least one area that requires more research.

Societal, political and the resulting economic pressures are demanding higher reliabilities from the warm and hot structures. This has already come to pass in such safety conscious power devices as the nuclear reactors and in such strategic and expensive vehicles as the space shuttle and the B-1 aircraft. In particular, we are referring to the concept of fracture mechanics design of structures. This design approach is based on the valid premise that current levels of proficiency in either materials processing or nondestructive quality control are not sufficient to preclude flaws or cracks in structural components, and indeed, it is the rate of growth of these flaws under fatigue or creep loading conditions that determines the useful lives of the components.

Given that this fracture mechanics approach will become widely accepted and mandated, the question is then whether we metallurgists are ready to incorporate, say, fatigue crack growth resistance and creep crack growth resistance into superalloy design. The answer to this question, unfortunately, is not an encouraging one. Fatique crack growth behavior of superalloys has received very little attention. $(1-3)$ Despite the voluminous empirical activity on steels, the current understanding even for these high tonnage alloys is at best phenomenological, e.g., Refs. 4-6. The situation with respect to crack growth under creep conditions is even more primitive. There has been only one systematic study on the crack growth behavior of superalloys under creep loading canditions $(7)$ and a few on steels $(8,9)$, but here again no clear mechanistic understanding exists.

It is evident that, for materials in general and superalloys in particular, the major stumbling block in understanding flaw growth resistances is the lack of basic theories from which one could identify at least the dependence of these composite properties on the more well understood materials properties such as yield strength, ductility, creep strength, etc. Such theories, when verified or modified by critical experiments can serve as the bases for costeffective, scientific alloy design.

In this paper, we present our attempts at developing these theorles and their uses to identify tentative alloy design guidelines for flaw growth resistance in superalloys so that a better basis for alloy design can be developed 


\section{FATIGUE CRACK GROWTH RESISTANCE}

The history of the application of fracture mechanics to fatigue crack propagation is not a long one. Its origin can be traced to the work of Paris in 1964.(10) A good source for the terminologies, test specimen design, test procedures and other details of experimental fracture mechanics can be found in Refs. (11) and (12). In this section we present a fast compaction of exisling knowledge calegorized in terms of trends in malerials behavior. As will become evident, fatigue crack growth behavior in nickel-base superalloys appears different from that in cobalt-base alloys and from that in ductile steels and aluminum alloys. In order to highlight these differences, the fatigue crack growth theories will be derived in some detail.

\section{Brief Compaction of the Fatigue Crack Growth Literature}

Fatigue crack propagation data are usually presented in the form of a loglog plot of per cycle crack propagation rate da/dN versus the stress intensity range* $\Delta \mathrm{K}$, which is equal to the applied cyclic stress range $\Delta \sigma$ intensified by the square root of the crack length a. In ductile materials such as tough steels and the weaker aluminum alloys, in which crack growth is noncrystallographic, the typical da/dN versus $\Delta K$ plot is schematically shown in Fig. 1. There is an initial threshold regime I where da/dN rapidly increases with $\Delta K$. The existence of this threshold behavior is of significant practical importance since it represents a "fail-safe" limit in the fatigue growth of cracks. This threshold regime is followed by regime II where da/dN is less sharply dependent on $\Delta K$. At still higher $\Delta K$ levels, da/dN increases very rapidly as the stress intensity approaches the fracture toughness $K_{I C}$ of the material. $K_{I C}$ and the alloy design aspects of this parameter are discussed in some detaih elsewhere. $(13,14)$ The second regime or the so-called Paris law regime(10) has been most extensively studied. In tough steels and aluminum alloys, crack propagation in this regime is ductile, noncrystallographic, and without such brillle modes as cleavage or intergranular cracking occuring in a superlmposed manner ahead of the crack front. In these cases, da/dN is empirically related to $\Delta K$ by the Paris power law correlation

$$
\mathrm{da} / \mathrm{dN}=A(\Delta K)^{B}
$$

The crack propagation rate in this regime for the ductile alloys is found to be sensibly independent of microstructural details as well as alloy systems. This insensitivity to microstructure is vividly shown in Fig. $2,(4,6)$ where a host of tough and ductile steels, distinctively different in many respects, are found to behave similarly during fatigue crack growth. The da/dN of ductile alloys is also found to be insensitive to fatigue frequency, and sensibly independent of the stress ratio $R$, which is the ratio of the minimum stress intensity level, $K_{m i n}$, and the maximum stress intensity level, $K_{\max }$. The distinctive fcature of the ductilc alloys is that their da/dN's are related to $\Delta K$ by a power exponent $B$ of around 2, see for example the behavior of tough steels, Fig. 2 .

On the other hand, when brittle modes are found to superimpose onto the ductile noncrystallographic cracking mode, the power law exponent B in Eq. (1) is found to vary from 2 to 6 depending on the stress ratio $R$. Temper-embrittled steels $(6)$ and the non-planar slip, cobalt-base superalloys $(2)$ may be examples of this behavior.

* Latest fracture mechanics formulisms refer to an effective stress intensity range ( $\Delta K_{e f f}$ ) which is $\Delta K$ modified by crack closure considerations. (38) Detailed mechanistic understanding of crack closure is not available at present and $\Delta \mathrm{K}_{\text {eff }}$ is experimentally measured. However, the arguments presented in this paper are not significantly affected by crack closure. 


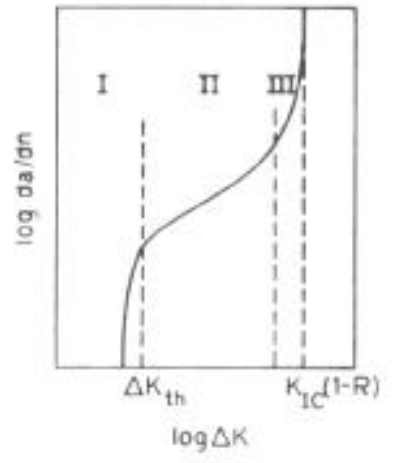

Fig. 1. Typical schematic of often observed da/dN versus $\Delta \mathrm{K}$ behavior of ductile material, showing drop-off of da/dN at low $\Delta K$ levels.

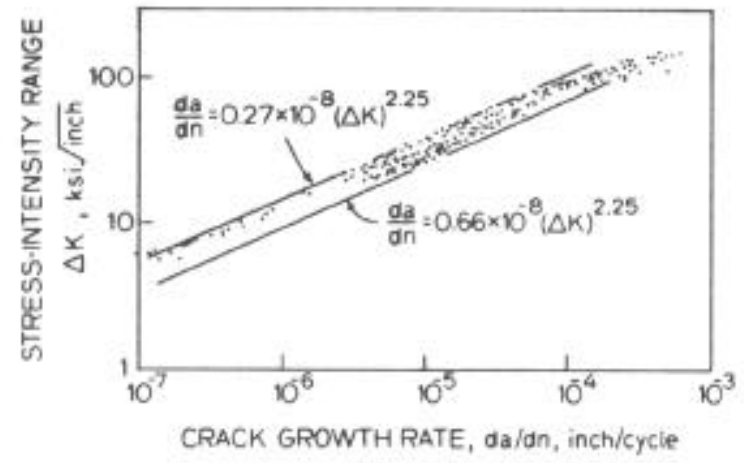

Fig. 2. Fatigue crack propagation behavior of a variety of tough steels, showing the apparent insensitivity of da/dN to microstructural variations. $(3-6)$

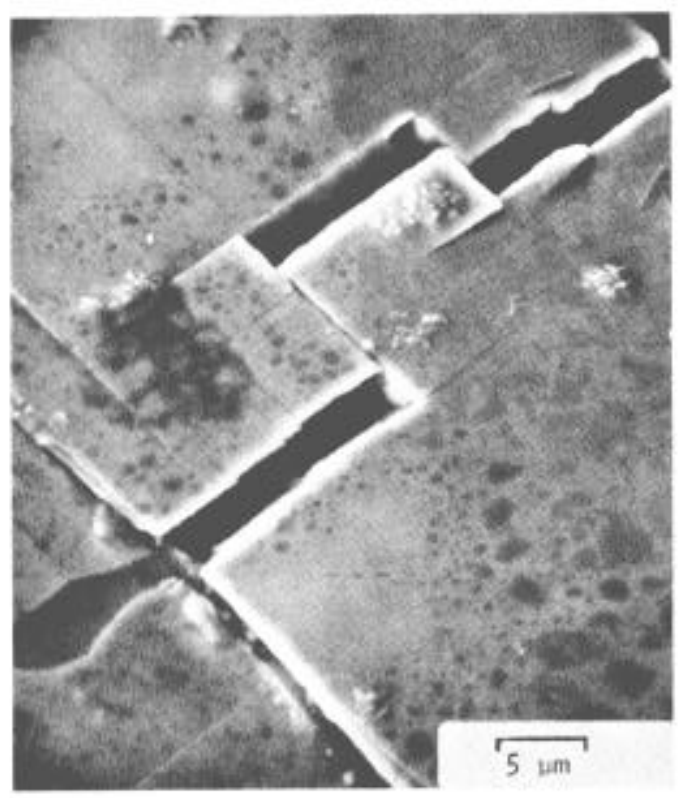

Fig. 3. Crystallographic slip band cracking in a monocrystalline nickel-base superalloy tested in high frequency $\mathrm{fa}^{-}$ tigue. Ref. (3).

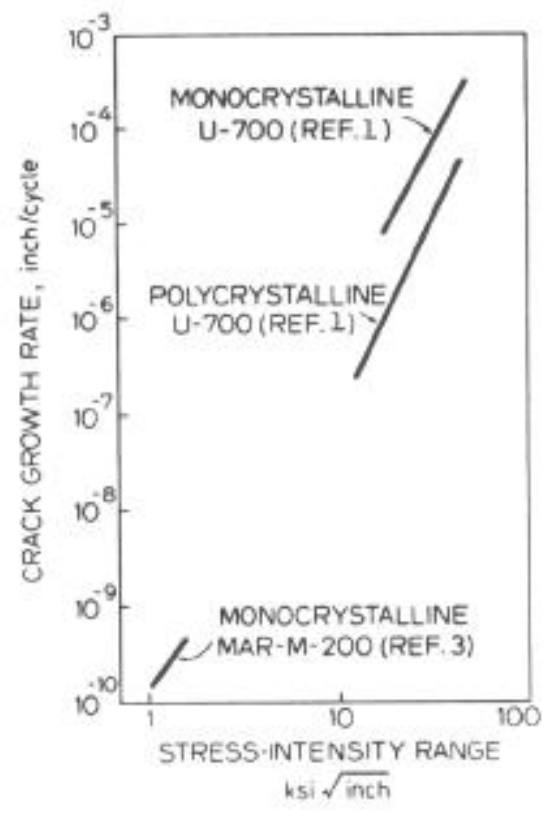

Fig. 4. Fatique crack propagation behavior in nickelbase superalloys at high(1) and low (3) $\triangle K$ levels. No drop-off in da/dN at low $\Delta K$ levels is apparent. 
The planar slip, nickel-base superalloys fall into yet another class of behavior. Limited studies show that fatigue cracks propagate in nickel-base superalloys along the crystallographic slip planes, $(1,3,15,16)$ see for example Fig. 3; and it is found that this crystallographic constraint manifests in a crack growth power law exponent that is close. to 4 over a wide range of $\Delta K$ levels(1,3) Fig. 4. In fact, it appears that the threshold behavior, which characterizes steels and other alloys, may not be present in the only superalloy (Mar-M200) that was investigated at the very low $\Delta K$ levels. (3)

From the above compacted review, it is clear that there are at least four major aspects of fatigue crack propagation that need to be modeled. These are (a) derivation of crack propagation rates in the Paris law regime or regime II; (b) the threshold transition behavior; (c) the effect of superimposed brittle modes; and (d) the effects of crystallographic constraints on crack growth behavior. Mandate (a) and especially (d) are of particular interest to superalloys. The understanding of Mandate (b) is also extremely important, especially since there is that suspicion that threshold or "fail-safe" behavior may not occur in at least some superalloys. We will briefly discuss, but will not derive Mandate (c), since the modelling of superimposed rracture modes is mathematically very complex, if not impossible, at this time.

\section{Cont inuous Fatigue Crack Growth Theory (Noncrystallographic)}

Physically, this model is based on the self-evident failure criterion that the crack will advance into an element of cyclically deformed material in the plastic zone ahead of a crack when the strain in that element reaches or exceeds the fracture strain $\varepsilon_{F}$ corresponding to the state of stress prevailing ahead of the crack tip in that material.

$$
\varepsilon(x) \geq \varepsilon_{F} \begin{aligned}
& \text { for failure of the general element at a } \\
& \text { distance } x \text { from the crack tip }
\end{aligned}
$$

This $\varepsilon_{F}$ can be approximated by plane strain ductility when the plastic zone sizes are much smaller than the thickness of the specimen or the component, while it would be close to the uniaxial tensile ductility when the stress state is one of plane stress, as in cases where plastic zone sizes are comparable to the specimen or component thickness. It should be mentioned here that in most structural applications (except in some thin aircraft body structures) the plane strain condition would be expected ahead of a crack lip and, Llius, plane strain ductility is an important property with respect to flaw growth as implied by our failure criterion, Eq. (2).

Having defined a failure criterion, it is then necessay to identify the strain distribution ahead of the crack tip so that one can in turn identify the region where the failure criterion will be satisfied and into which the crack will advance. For this purpose we use a strain diseribution due to Rice, (12) modified to account for crack tip biunting due to cyclic plastic flow at the crack tip, where

$$
\varepsilon(x)=\left[2 \varepsilon_{y, c} f(R, \Delta k)\right] /\left[4 \pi \sigma_{y, c}{ }^{2}(1+n)(x+\rho(R, \Delta k))\right]^{1 / 1+n}
$$

where

$$
f(R, \Delta k)=\left(2 k_{\max }{ }^{2}\right)^{1 / 1+n}-\Delta k^{2 / 1+n}+\Delta k^{2 / 1+n}
$$

Again, $K_{\text {max }}$ is the maximum stress intensity factor; $R$ is the ratio of the minimum to the maximum stress intensity factors, that is, $K_{m i n} / K_{m a x} ; \Delta K$ is the stress intensity range which equals $K_{\max }-K_{\min }$; $\Delta k$ is the instantaneous level of cyclic stress intensity which is defined as the difference between the instantaneous level of stress intensity $k$ and the minimum stress intensity $K_{m i n}$; 
$\rho(R, \Delta k)$ is the crack tip radius indicative of the crack tip blunting; $E_{y, c}$ is the ratio of the cyclic yield stress $\sigma_{y, c}$ to the elastic modulus $E$, and $h$ ' is the cyclic work hardening exponent. The loading parameters described above are defined graphically in the schematic Fig. 5.

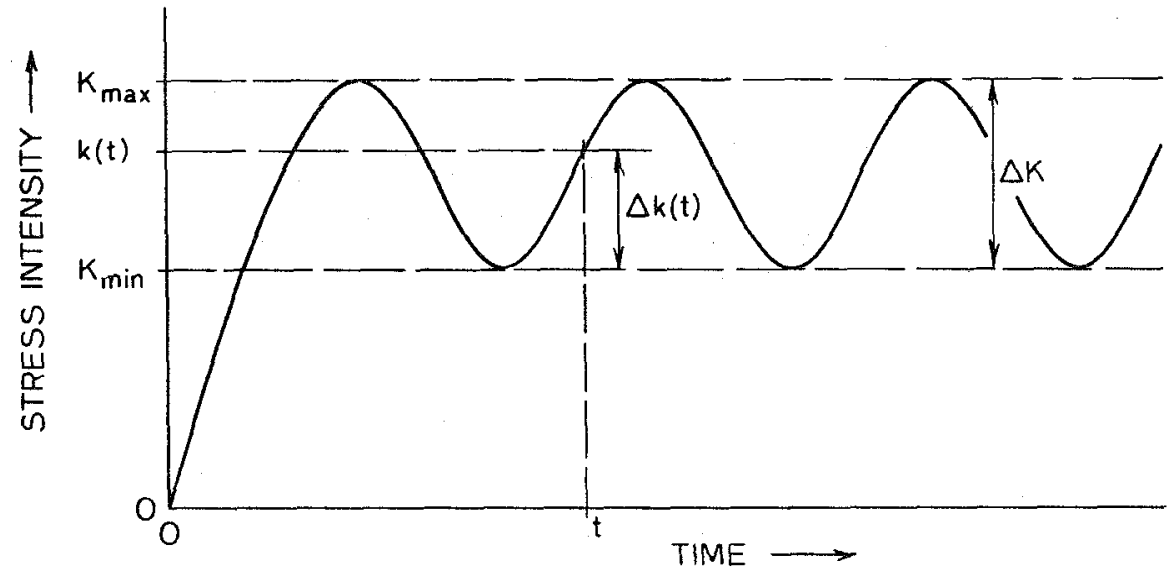

Fig. 5. Schematic of a fatigue loading curve defining the loading parameters referred to in the text.

At any given instant of time $t$, when the instantaneous cyclic stress intensity is $\Delta k$, Eqs. (2) and (3) can be combined to allow for the calculation of a distance $\Delta a$ over which the failure criterion will be satisfied, i.e., $\Delta a r^{-}$ presents that increment of crack advance associated with $\Delta k$, where

$$
\Delta a=\left[\left(2 \varepsilon_{y, c} / \varepsilon_{F}\right)^{n+1}[f(R, \Delta k)] / 4 \pi \sigma_{y, c^{2}}(n+1)\right]-\rho(R, \Delta k)
$$

This distance is depicted in Fig. 6 for further reference.

The time it takes for the crack to advance by $\Delta a$ should be governed by the rate at which damage accumulates ahead of the crack tip. Since our failure criterion is strain controlled, in our model damage is synonymous with the level of plastic strain ahead of the crack tip. Hence, we can infer that the damage accumulation rate would be proportional to the local plastic strain rate. Further, for a continuous crack propagation process, it is necessary that the strains in the elements of material at distances $x>\Delta a$ from the crack tip increase to new and higher levels, as indicated by the dotted curve in Fig. 6, appropriate to the new crack tip position.

Combining these two aspects it can be concluded that for continuous ductile noncrystallographic crack propagation the time duration for the crack tip advancement, at any given $\Delta k$, is equal to the time it would lake for the strain field to readjust (or the damage to build up) in the region ahead of the crack tip. This time, $\Delta t(x)$, can be obtained by dividing the expected strain excur- 
sion $\Delta \varepsilon(x)$ by the local strain rate $\dot{\varepsilon}(x)$ both of which are dependent on distance from the crack tip. However, we are interested only in the maximum value of this $\Delta t(x)$ since it will be the rate controlling value in the strain readjustment process as well as the continuous crack growth process. The details of this calculation are purely algebraic and will not be shown here as they are already available in detail in our earlier publication. (17) It suffices to note that the maximum value of $\Delta t(x)$, say $\Delta t_{\max }$, can be obtained and

$$
\mathrm{da} / \mathrm{dt}=\Delta \mathrm{a} / \Delta \mathrm{t}_{\max }
$$

where da/dt is the instantaneous velocity of crack growth at a given cyclic stress intensity $\Delta k$.

We can now calculate da/dN or the per cycle crack propagation rate as

$$
\mathrm{da} / \mathrm{dN}=\int_{\substack{\text { loading } \\ \text { half cycle }}}\left(\Delta \mathrm{a} / \Delta t_{\max }\right) \mathrm{dt}
$$

Here, we presume that the crack does not grow during the unloading half cycle since the crack tip stresses are expected to be compressive. Again for the sake of brevity, we will skip the integration procedures involved in Eq. (6). Interested readers are referred to References (3) and (17) for the details. The final expression of $d a / d N$ for continuous, noncrystallographic crack propagation is

$$
\mathrm{da} / \mathrm{dN}=\Delta \mathrm{K}^{2} \mathrm{~g}(R) / \alpha \pi \sigma_{Y, c^{1-n_{E}}}^{1+n} \varepsilon_{F}^{1+n}(n+1)
$$

where

$$
g(R)=\left[2-\left[2^{1 / 1+n}-(1-R)^{2 / 1+n}\right]^{n+1}\right] /(1-R)^{2}
$$

The parameter $\alpha$ is a constant depending on the extent of crack tip blunting, but varies in value only between unity and $2 .(3,17)$

For the simple case where work-hardening can be considered negligible, or $n=0$, this equation reduces to

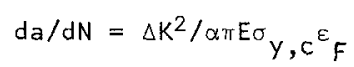

A direct numerical comparison of this theoretical rate with experimental properties data is not always possible, since the materials properties required are not only cyclic in nature but also those obtained under plane strain constraint. Neither cyclic, nor plane strain, and certainly not cyclic plane strain, data are readily available. However, for the present, we will briefly examine the functional aspects of the theoretical predictions with the available experimental data to verify the general validity of the theory.

The theory predicts a second power dependence of da/dN on $\Delta K$; a mild dependence of $d a / d N$ on stress ratio $R$ through the mild function $g(R)$, and that da/dN independent of test frequency. All these predictions are in agreement with experimental results on ductile noncrystallographic crack growth without superimposed brittle failure modes. $(4-6)$ Secondly, the apparent insensitivity of $\mathrm{da} / \mathrm{dN}$ to microstructural manipulations in this regime can also be explained through the theory. da/dN is predicted to be inversely proportional to the product of $E^{1+n_{\sigma_{Y}}, c^{1-n_{1}} \varepsilon_{F}{ }^{1+n} \text {, see } E q . ~(7) . * ~ W h i l e ~} E$ does not vary much with

* It should be mentioned here that resistance to low cycle fatigue which is crack propagation controlled in most engineering alloys should also similarly depend on these same properties. 


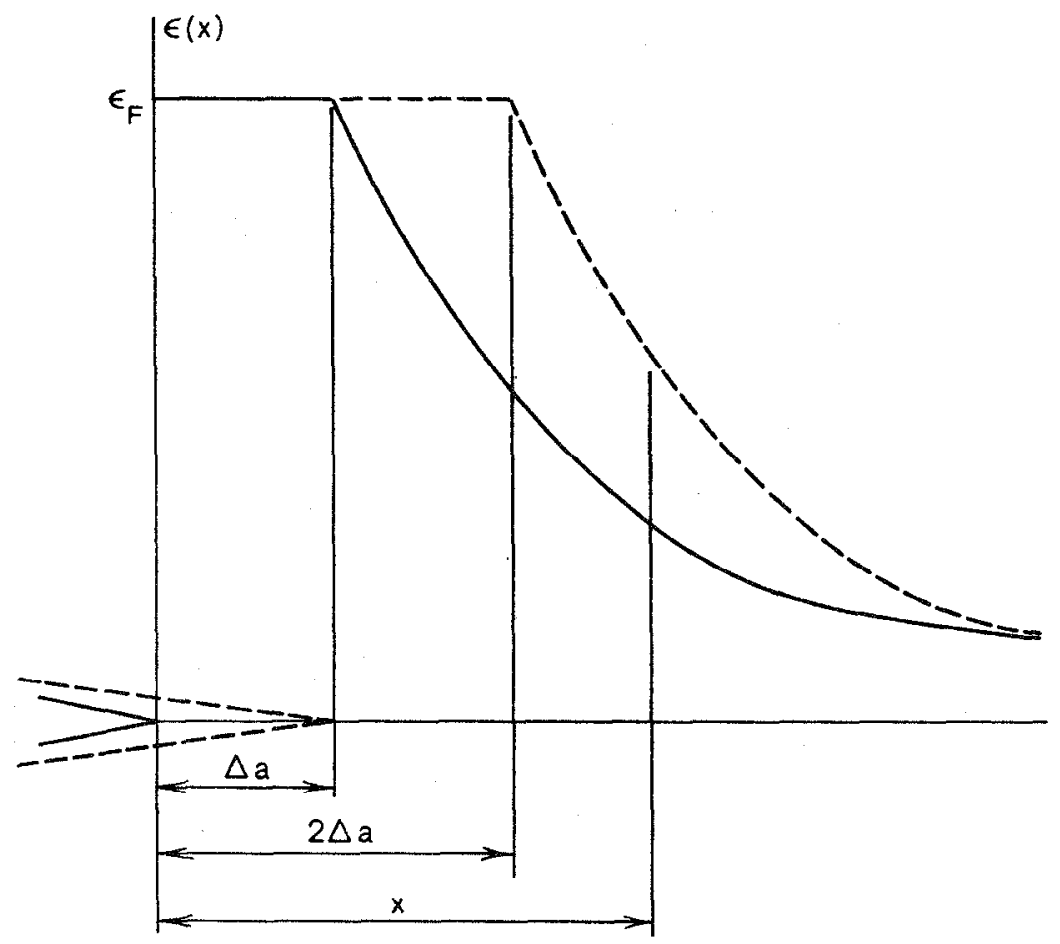

Fig. 6. Schematic showing crack advancing by $\Delta a$ as the strain field readjusts itself ahead of the crack.

microstructural manipulation of the hase alloy, it is well known that any attempts to strengthen a material lead to a loss in ductility $\varepsilon_{F} \cdot(14)$ The overall result is that da/dN which is predicted to be proportional to the product $\sigma_{y, c} 1-n_{\varepsilon_{F}}{ }^{+}+n$ tends to be insensitive to microstructural manipulation. This can but having different properties: the steel, $A 533-B$, which has a $E$ of $30 \times 10^{6}$ psi, a $\sigma_{y, c}$ of $60 \mathrm{ksi}$, a uniaxial ductility $\varepsilon_{F}$ of 1.6 , and a cyclic work hardening exponent of 0.12 ; and a highly strengthened $18 \% \mathrm{Ni}$ Maraging steel with a $\sigma_{y, c}$ of $200 \mathrm{ksi}$, a uniaxial ductility of 0.8 , a $E$ of $26 \times 10^{6} \mathrm{psi}$, and a cycllic work hardening exponent of 0.08 . (3) A calculation of the product $E^{1+n_{\sigma}}{ }_{y, c} c^{-n}$ $\varepsilon_{F} 1+n$ yields a value of $3 \times 10^{4}$ for the $A 533$ steel and a value of $6 \times 10^{4}$ for the Maraging steel. This comparison indicates that, despite the extreme difference in yield strength levels, the fatigue crack propagation resistance of these steels as reflected by the above product is quite close, mainly as a consequence of the corresponding lowering of the ductilities. Accordingly, the microstructural insensitive behavior depicted by Fig. 2 can be considered to be due to the perennial conflict between strength and ductility.

In the case of the less ductile materials where brittle failure modes such as cleavage and intergranular separation tend to occur in a superimposed manner ahead of the growing fatigue crack, and adding to the crack growth rate, the contribution due to the ductile noncrystallographic cracking mode can often be distinguished from the superimposed modes by the fatigue fracture surface features. The ductile contribution is reflected by the spacing of fatigue striations on the fracture surface and not surprisingly is often found to follow the predicted $\Delta K^{2}$ dependence. $(4-6)$ The overall macroscopic growth rate 
is characterized, however, by increasing B values with increasing stress ratio $R,(4-6)$ since contribution of superimposed brittle modes, which would depend mainly on the maximum local stréss levels, would be enhanced with increasing $R$ values. Thus, one can look upon the behavior of these less ductile materials as a ductile crack growth behavior modified by local variations in toughness of the microstructure due to the presence of weak grain boundaries, brittle inclusions, etc. The cobalt-base superalloy fatigue crack growth behavior (2) may indeed be a good example of this modified ductile case.

\section{Threshold Fat Igue Crack Growth}

We propose that the threshold fatigue crack growth behavior can be explained through energetics arguments. In particular, borrowing from phase transformation theories, $(18-21)$ we propose that whereas regime II represents continuous crack advance, regime I involves inhibited crack advance through the lateral motion of ledges on the crack front. Further, the extent of the inhibited regime will depend on the atomistic structure of the crack front, which is dependent on crystallographic constraints.

The first step in this analysis is to perform an energy accounting of the free energy change attendant to an incremental change in crack front position. For this purpose, we consider a crack front which is straight in the absence of an applied stress intensity but assumes a bowed configuration when a stress intensity range $\Delta K$ is applied, see dotted curve in Fig. 7. The length $L$ could be the fracture mechanical specimen thickness over which the crack front ex-

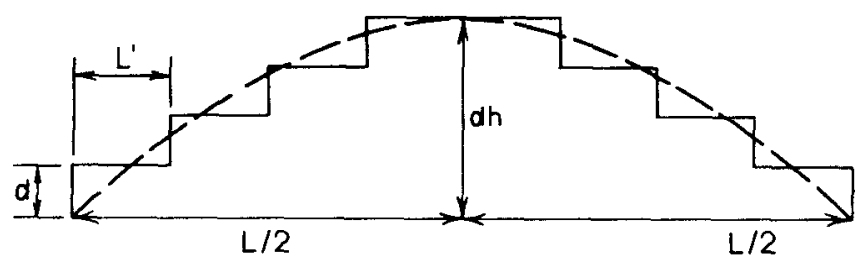

Fig. 7. Schematic showing the configuration of a noncrystallographic (continuous dotted curve) and crystal lographic (stepped solid curve) crack

fronts from the initially straight configuration.

tends, or a microstructural dimension such as grain size or mean inclusion spacing between which the crack front is constrained in a real system. For the sake of geometric simplicity the crack front is assumed to be a circular arc and the average crack advance due to the bowed out configuration is taken to be $d a=d h / 2$, see the dotted curve in Fig. 7. The incremental free energy change, $\delta F$, due to this bowing of the crack front can be written as

$$
\delta F=-\left(\Delta K^{2} / \pi E\right) L d a+\left[d\left(A_{s} \gamma_{s}\right) / d a\right] d a+[d(\Gamma S) / d a] d a+\left[d\left(V_{\gamma p}\right) / d a\right] d a
$$

The first term in the right hand side of Eq. (9) is the driving free energy due to the loading stress intensity range $\Delta K$ and represents the decrease in 
the potential energy of the system due to a decrease in specimen compliance resulting from the crack advance da. The second term is the increase in surface free energy due to the creation of new fracture surface area where $A_{s}$ is the area of the fractured cross section and $\gamma_{s}$ is the specific surface fres energy. The third term is the increase in the line energy of the crack front due to the increase in the length $S$ where $\Gamma$ is the line energy per unit length of the crack front in analogy with a line tension of line defects such as dislocations. The last term is the increase in the plastic strain energy due to the crack growth where $\gamma_{p}$ is the strain energy per unit volume and $V$ is the critical volume near the crack front in which this increase occurs. We now recast $\mathrm{Eq} .(9)$ as

$$
\delta F=\left[-\left(\Delta K^{2} L / E_{\pi}\right)+\gamma_{S}\left(d A_{s} / d a\right)+\Gamma(d S / d a)+s(d \Gamma / d a)+\gamma_{p}(d V / d a)\right] d a
$$

$A_{s}$ can be taken to be equal to $L d h$, and hence $d A_{S} / d a$ is nearly equal to $2 L$. Similarly, $S$ can be approximated by $\sqrt{L^{2}+4 d a^{2}}$ and thus $d S / d a$ can be approximated by 4da/L. Further, in the fourth term of $\mathrm{Eq} .(10)$, we allow for the variation of the 1 ine tension $\Gamma$ with $a$, or in our case with crack front curvature. We will come to the importance of this term when we consider the effect of crystallographic constraints. In the last term, $\gamma_{p}$ can be approximated by $\sigma_{Y} \varepsilon_{F}$. Further, the volume $V$ can be viewed as $\frac{1}{2} A C O D$, where $C O D$, the crack opening displacement, reflects the lineal dimension normal to the plane of the drawing, Fig. 7, over which the plastic energy $\gamma_{\text {pas }}$ to be enhanced. Hence, dV/da can be approximated by $\left[\Delta K^{2} / 2 \pi E \sigma_{y, c}\right]$. Substituting these values into Eq. (10), we get

$$
\delta F=\left[-\left(\Delta K^{2} / \pi E\right) L+2 \gamma_{s} L+\Gamma 4 d a / L+4 S(d \Gamma / d a)+\left(\Delta K^{2} / 2 \pi E\right) \varepsilon_{F} L\right] d a
$$

Eq. (11) is already in incremental form, hence the critical driving free energy $\delta F *$ (which will correspond to a $\Delta K *$ ) which must be exceeded in order for the crack to advance normally can be obtained by letting $\delta F=0$. This results in

$$
(\Delta K *)^{2}\left(2-\varepsilon_{\mathrm{F}}\right) / 2 E \pi=2 \gamma_{\mathrm{S}}+4 \Gamma \mathrm{da} / \mathrm{L}^{2}+(\mathrm{d} \Gamma / \mathrm{da}) 4 S / L
$$

The second term in the right hand side is usually small due to the da/L ${ }^{2}$ term and can be neglected. For further analysis Eq. (12) then becomes

$$
\Delta K^{*}=\left[\left(2 E \pi / 2-\varepsilon_{F}\right)\left(2 \gamma_{s}+(d \Gamma / d a)(4 S / L)\right)\right]^{\frac{1}{2}}
$$

The last term in Eq. (13) depends on $\mathrm{d} \Gamma / \mathrm{da}$ and for a noncrystallographic crack front it will be negligible since $d \Gamma / d a \simeq 0$; that is in analogy to a random or disordered grain boundary where there are no preferred low line tension orientations for a noncrystallographic crack front or boundary. Accordingly, for a noncrystallographic crack front, we maintain that the threshold $\Delta K$ is

$$
\Delta \mathrm{K}_{\mathrm{n}} *=\left[4 \mathrm{Err}_{\mathrm{S}} /\left(2-\varepsilon_{\mathrm{F}}\right)\right]^{\frac{1}{2}}
$$

When $\Lambda K \leq \Delta K *$, i.e., in regime $I$, the crack front is in metastable equilibrium with the driving force and in analogy with the generalized theory of boundary motion, (15) the crack cannot move normally, but could move only through the lateral motion of ledge or step on the crack front, with the crack advancing by one ledge height as that ledge passes across the crack front. The crack growth rate through such a mechanism would depend on the number of ledges on the crack front and the velocity of the average ledge. In a perfect material the crack front can develop a ledge by a nucleation process in the presence of the applied $\Delta K$. If $N_{1}$ is the number of ledges per unit length of the crack front formed by this nucleation process and $d$ is the ledge height in the direction of crack growth, the effective growth rate of the crack front in regime I would then be 


$$
(\mathrm{da} / \mathrm{dN})_{\mathrm{I}, \mathrm{n}}=(\mathrm{da} / \mathrm{dN})_{\text {ledge }} \mathrm{d} \mathrm{N}_{1}
$$

$(\mathrm{da} / \mathrm{dN})_{\text {ledge }}$ can be estimated by knowing the local $\Delta K$ near a ledge. A recent analys is on local $\Delta K^{\prime}$ s near perturbations on a crack front shows that, depending on the perturbation geometry, the local stress intensity near the perturbation could be about a factor of two larger than the nominal leve1. (22) Representing this local value by $\beta \Delta K$ where $\beta$ is this magnifying factor, (32) allowing (da/dN) ledge to be that of a noncrystallographic fatigue crack, and using the relationship that the number of nucleated ledges on the crack front is proportional to $\Delta K^{2} \exp \left(-D / \Delta K^{2}\right)$ as derived in detail elsewhere, $(3)$ we obtain

$$
(\mathrm{da} / \mathrm{dN})_{I, \mathrm{n}}=C \Delta \mathrm{K}^{4} \exp \left(-\mathrm{D} / \Delta \mathrm{K}^{2}\right)
$$

where C and D are material constants which can be found in Ref. (3).

It is clear from Eq. (17) that the functional dependence of noncrystallographic, regime $I d a / d N$ on $\Delta K$ below $\Delta K_{n} *$ is very sharp through the $\exp \left(-0 / \Delta K^{2}\right)$ term.

This prediction of the sharp and falling functional dependence of da/dN on regime I crack growth, as $\Delta K$ dccrcases, is indeed consistent with observations, say, in steels and some aluminum alloys where crack growth is noncrystallographic and where data are avaliable, see the literature survey in Ref. (3) and the schematic representation in Fig. 1. Further, the predicted transition $\Delta \mathrm{K}_{\mathrm{n}}{ }^{*}$, see Eq. (14), is in agreement with the experimental threshold $\Delta \mathrm{K}$ results, when one allows for crack closure considerations. (3) It appears that these equations should also apply in a similar fashion to nonplanar slip superalloys, e.g., the cobalt-base al loys, which are cxpected to show noncrystallographic crack growth. But there are no data on the low $\Delta K$ regime crack growth behavior of this class of alloys; more on this aspect later.

With regard to structural alloy design in general, it appears from Eq. (14) that threshold $\Delta K$, i.e., fail-safe $\Delta K$ can be raised if one can raise the elastic modulus, surface energy and fracture strain. The first two quantities are more or less fixed by the system, and hence, it appears that a high $\varepsilon_{F}$ is important not only for $d a / d N$ resistance in the Paris regime, but also with respect to raising the threshold $\Delta K$.

\section{Planar Slip Superalloy Fatigue Crack Growth}

If the crack front is crystallographic, as in the case of the planar slip nickel-base superalloys $(1,3)$ then the effect of crystallographic constraints can become very important in the transition analysis. In this case the crack front $l$ ine tension $\Gamma$ can be dependent on orientation, in the same manner as surface energy $\gamma_{s}$ which is known to be orientation dependent, see for example Ref. (21). In fact, it can be shown that a crack front that is growing in the $<110\rangle$ close packed direction and lying in the close packed $\{111\}$ plane, which are the slip directions and planes of the fcc nickel-base alloy, is itself in a close packed orientation, and hence, in an energy cusp orientation. Accordingly, for this category of crystallographic crack fronts one would expect $\mathrm{d} \Gamma / \mathrm{da}$ in Eq. (13) to be very large, approaching infinite values when the energy cusp is sharp. Cahn has estimated that surface energy cusps in crystalline solids can be on the order of $10^{8}$ times $Y_{5}$. (18) Hence, dr/da can accordingly be $10^{8}$ or so times $\Gamma$. From Eq. (13), and neglecting all terms but that containing $d \Gamma / d a$, and approximating $S \simeq L$, we $f i n d$ that the threshold $\Delta K$ for for crystallographic cracks is

$$
\Delta K_{c} *=\left[\left(8 E_{\pi} /\left(2-\varepsilon_{F}\right)\right)(d \Gamma / d a)\right]^{\frac{1}{2}}
$$

Also as a consequence of a high $d \Gamma / d a$, the crystallographic crack front will avoid a continuously bowed configuration. Instead, it would be energetically 
more probable for the front to achleve the bowed configuration through a distribution of ledges, see solid lines. in Fig. 7. In this microscopically stepped configuration the edges of the steps will contribute some additional cnergy, but, being non-cusp oriented, the added energy will be much less than if the entire front is continuously curved.

In such a configuration, the crack can move by the lateral motion of the steps under the action of the applied $\Delta K \leq \Delta K{ }^{*}$. The effective crack propagation rate normal to the crack front, $(\mathrm{da} / \mathrm{dN})_{\mathrm{I}, \mathrm{c}}$, due to this lateral growth mechanism with existing steps would then be

$$
(\mathrm{da} / \mathrm{dN})_{I, c}=(\mathrm{da} / \mathrm{dN})_{\text {ledge }}\left(\mathrm{d} / \mathrm{L}^{\prime}\right)
$$

where $(d a / d N)$ ledge is again the lateral crack growth rate per cycle and $d$ and $L^{\prime}$ are the step height and spacing, respectively, see Fig. 7. Again, (da/dN) ledge can be approximated by the da/dN expression of a noncrystallographic lecgack front, i.e., Eq. (7), with the $\Delta K$ chosen as the local $\Delta K$ near the ledge edge. As before, we can represent the local stress incensily range ahead of a ledge by $B \Delta K$ where $\beta$ is a parameter larger than unity but usually smaller than 2 .

The values of $d / L^{\prime}$ in $E q .(18)$ are more difficult to estimate. In this paper, we restrict ourselves to an upper limit estimate of this ratio. For this purpose we can assume that $d$ would be a multiple of the lattice spacing normal to the crack front--a reasonable estimate being the width of a slip band. Further, assuming that the ledges are equally spaced, the smallest interledge spacing that can be supported at a given $\Delta K$ level would be the critical ledge nucleus size that can be supported by $\Delta K$, where $(3)$

$$
L^{\prime}=2 E \gamma_{S} L \pi /\left(2-\varepsilon_{F}\right) \Delta K^{2}
$$

Combining Eqs. (18) and (19), we obtain

$$
(d a / d N)_{I, c}=\left[d g(R) /(\alpha \pi)^{2} \gamma_{s} L E^{2+n} \varepsilon_{F}^{n}{ }^{n} y, c{ }^{1-n}\right] \Delta K^{4}
$$

Thus, Eq. (20) predicts a fourth power dependence of da/dN on $\Delta K$ for crystal lographic cracking when $\Delta K<\Delta K^{*}$, and $\Delta K_{*} *$ is expected to be very large. In fact, taking $d \Gamma / d a$ to be $10^{8} \mathrm{~T}$ and $\Gamma$ to be $\gamma_{5}$ (COD). and using $E$ of $20 \times 10^{6} \mathrm{psi}$, $\varepsilon_{F}$ of 0.2 , we estimate $\Delta K_{c} *$ to be $150 \mathrm{ks}$ i $\sqrt{\mathrm{in}}$ for nickel-base superalloys.

The significance of this high value for $\Delta K_{c} *$ is best seen if $\Delta K_{c} *, \Delta K_{n} *$ and $K_{I}$ are discussed together. $\Delta K_{n} *$ being less than $K_{\text {I }}$ divides the $\mathrm{da} / \mathrm{dN}$ Gehavior of the ductile, noncrystallographic crackfng alloys into the two regimes, as shown in Fig. 1, where below $\Delta K_{n} *$ the da/dN drops off exponentially with decreasing $\Delta K$, consistent with our theory in the previous section. For the crystallographic cracking or nickel-base superalloy case, the $\Delta K_{c} *$ value calculated is indeed greater than even $K_{I C}$, which is quoted to be $50^{c} \mathrm{ksi} \sqrt{\mathrm{in}}$ for superalloys. This means that we are predicting that the da/dN behavior of this type of alloy will not exhibit any drop-offs, but will follow $\Delta \mathrm{K}^{4}$ behavior throughout the entire $\Delta \mathrm{K}$ range. This indeed may be the case, since $\Delta K^{4}$ dependence is seen at higher $\Delta K^{\prime} s$ for a host of $n i c k e l$-base superalloys, and it was also observed with no fall-off evident in the one case where the da/dN behavior of a nickel-base superalloy was investigated down to a low $\Delta K$ of $1 \mathrm{ksi} \sqrt{\mathrm{in}}$, see Fig. 4. The dependences of the predicted crystallographic crack growth rate on the various properties are similar to those derived for the noncrystallographic case; this can be seen by comparing Eq. (20) with Eq. (7). 
Pre-existing flaws can also propagate under creep loading conditions. The importance of creep crack propagation was not realized until a few years ago $(7-9)$ and we are not aware of any theoretical model for this mode of crack growth that is suitable for alloy design uses. In what follows, we will derive an approximate model which is fairly consistent with the scant experimental data and which may be helpful in providing alloy design guidelines.

Physically, this model assumes that the crack will advance as a consequence of stress rupture ahead of the crack caused by enhanced creep rates due to the high local crack tip stresses. We estimate the crack tip stress o using the Orowan-Irwin elastic approach as

$$
\sigma=\sigma_{a} \sqrt{a / p}
$$

where $\sigma_{a}$ is the applied nominal stress, a is the crack length and $\rho$ is the effective crack tip radius which depends on the extent of crack tip biunting. It should be noted that the elastic description of the crack tip stresses is only a first order approximation to the actual state of stress near the crack tip since creep deformation in this region can relax the local stresses.

The creep rate at this local stress level can be obtained using a phenomenological creep equation(14)

$$
\begin{aligned}
\dot{\varepsilon} & =A\left[\left(\sigma(x)-\sigma_{i}\right) / E\right]^{m} \exp \left(-Q_{c} * / R_{g} T\right) \\
& =A\left[\left(\sigma_{a} \sqrt{a / p}-\sigma_{j}\right) / E\right]^{m} \exp \left(-Q_{c} * / R_{g} T\right)
\end{aligned}
$$

where $A$ is a materials constant, $\sigma_{i}$ is the internal stress and can be insignificant compared to the high $\sigma(x), m$ is the stress exponent and $Q_{C} *$ is the activation energy of steady state creep and $R_{\mathrm{g}} T$ has the usual meaning.

The stress rupture time $t_{r}$ for an element undergoing creep can be obtained from the pseudo-basic equation relating $\dot{\varepsilon}(x)$ and $t_{r},(23,24)$

$$
t_{r}(x)=B /(\dot{\varepsilon}(x))^{\alpha}
$$

where the exponent $\alpha$ and the coefficient $B$ are material constants. This equation has a unique physical significance when $\alpha$ is equal to one in that it reflects a strain controlled failure criterion for stress rupture where the critical strain for failure is B, which would be a function of the stress rupture ductility. Experimentally, $\alpha$ is found to be on the order of 0.7-0.8 for superalloys $(23,24)$ and it appears from a recent study $(24)$ that $\alpha$ values less than unity may be related to the duration of and the strain during tertiary creep.

Substituting Eq. (22) into (23) and allowing $\sigma_{\text {; }}$ to be negligible, replacing $\sigma_{a} \sqrt{a}$ by $K$ the stress intensity factor, and differentlating Eq. (23) with respect to $x$ at the crack tip or $x=0$, and regrouping we obtain the creep crack growth rate expression

$$
\dot{a}=\left.\left(\mathrm{dx} / \mathrm{dt} \mathrm{t}_{r}\right)\right|_{\mathrm{x}=0}=\left[2 \mathrm{~A}^{\alpha} / \mathrm{Bm \alpha}^{\mathrm{m} \alpha} \rho^{\mathrm{m \alpha} / 2}-1\right] k^{m \alpha} \exp \left(-\alpha Q_{c} * / R_{g} T\right)
$$

The predictions of the above equation are functionally consistent with the experimental data on nickel-base superal loys $(7)$ and steels. $(8-9)$ Floreen, (7) for example, observed that, for several superalloys, the temperature dependence of $\dot{a}$ is through an activation energy of about $45 \mathrm{kcal} / \mathrm{mole}$ which is about 0.7 to 0.8 times that for steady state creep and the $K$ dependence is through an exponent smaller than the steady state creep stress exponent $m$. These observations are consistent with our predictions that the activation energy of creep crack growth should be the product of $\alpha$, which is $0.7-0.8$ for superalloys, 
times the activation energy for steady state creep which is around $66-76 \mathrm{kcal} /$ mole. Secondly, since $\alpha$ is less than unity, the exponent ma is expected to be slightly less than unity, again in agreement with Floreen's observations.

From Eq. (24), it appears then that all considerations that will reduce steady state creep rate and increase stress rupture ductility (higher $B$ values in Eqs. (23) and (24) would improve creep crack growth resistance. This again highlights the perennial conflict between strength--in this case, creep strength and ductility--or stress rupture ductility. For example, adding fine strengthening particles to a system, for example, the throia in TD-Nickel, the $\gamma^{\prime}$ in the nickel-base superalloys, no doubt decreases creep rates, but it also sharply reduces stress rupture ductility. (14)

The derived equation also predicts an inverse dependence of a on the crack tip radius $p$. A lower limit for $p$ is the fracture mechanics COD. This is because at higher temperatures it is reasonable to expect $\rho$ to relax to blunter configuration due to creep relaxation. The exact expression for $p$ is indeed what is required to convert the assumed elastic stress distribution, Eq. (21), to a more relaxed stress distribution ahead of a crack that is advancing under creep conditions. $\rho$ will certainly be a function of stress and temperature. It may also be affected by microstructural features in that such inhomogeneities as grain boundaries and inclusions can result in alterations of $\rho$.

Finally, although Eq. (24) is basically in terms of creep and stress rupture properties, it would be difficult to rank a resistance by ranking these other properties. This is because again the properties of interest would be those obtained in plane strain conditions and not the more readily available uniaxial or plane stress properties. Like the fatigue crack growth case, the only time plane stress is the condition ahead of the crack tip would be when the crack is huge--a situation that is not intentionally courted in practice.

\section{DISCUSSION}

The foregoing analysis of fatigue crack growth behavior can be summarized for alloy design purposes by the following expression agglomerated from. Eqs. (7), (8) and (20) and Eqs. (14) and (17), all of which were derived from first principles:

$$
\begin{gathered}
\mathrm{d} a / d N=\Delta K^{B} / Y \sigma_{y, c}{ }^{1-n} \varepsilon_{f}^{p_{E} q}(n+1) \gamma_{s}{ }^{r} \\
\Delta K_{n}^{*}=\left[4 E_{\pi} \gamma_{s} /\left(2-\varepsilon_{F}\right)\right]^{\frac{1}{2}} \\
\Delta K_{c} *>\Delta K_{n} *
\end{gathered}
$$

where $Y$ is a geometrical constant. In the absence of the usual wealth of empirical information which unfortunately is the case for superalloys, these equations provide at least the basis for discussion of the (anticipated) fatigue crack propagation behavior in superalloys. Undoubtedly, the points that we will be making can be viewed by prudent individuals, us included, as merely proposals for future experimental confirmation or denial. We will lead the cheering section if indeed the experimental verdict is one of denial for some of the predictions below.

Firstly, there appear to be three broad categories of fatigue crack growth behavior. The ductile noncrystallographic crack propagation mode which is characterized by $B$ of $2, p$ and $q$ of $(1+n)$ and $r$ of 0 in Eq. (25). By the way $n$ is usually between 0 and 0.2 . $(14,26)$ This ductile mode is always associated with a well defined threshold or "fail-safe" behavior where the crack growth rate falls off rapldly below $\Delta K_{n} *$. The second category is where superimposed brittle fracture modes occur ahead of the main crack leading to $B$ values varying anywhere from 2 to as high as 10 , depending on the stress ratio. 
A quantltative description of this mode is not available but it can be viewed as the ductile mode described above overshadowed by additional brittle modes promoted by decreases in toughness of the local microstructure ahead of the crack. Threshold behavior should also be present in this category but would not be as predictable in $\Delta \mathrm{K}_{\mathrm{n}} *$ as a consequence of variations of, say, the ductility $\varepsilon_{F}$ (or toughness) on a local scale. The third category is that where crack propagation is crystallographic occurring along slip planes and is characterized by $B$ of 4 , $p$ of $n$, $q$ of $(2+n)$ and $r$ of 1 in Eq. (25). In this category the threshold level is $\Delta \mathrm{K}_{*}^{*}$, Eq. (17), and is expected to be very high with the result that the $\Delta K^{4}$ Gehavior should indeed persist over all practical $\Delta K$ levels. Hence, in this crystallographic category we would not expect any sharp fall-off of crack growth rates. Schematically, the first and second categories follow the trends shown in Fig. 1 and the third or crystallographic category is expected to be that as shown in Fig. 4.

of these three categories of behavlor, the first one is the least insidious, since it exhibits a predictable $B$ value combined with a well-defined fail-safe $\Delta K$ level, whereas the second one is plagued by erratic $B$ values and fail-safe levels which could vary from batch to batch due to microstructural inhomogenieties. Even though the third category exhibits a predictable $B$ value, it can still be worrisome because we do not expect it to show a fail-safe behavior. Accordingly, it is unfortunate that only the second and third categories have been observed in superalloys. In the one carbide strengthened cobaltbase superalloy study reported to date, 2 the overall crack growth rate was characterized by a B value of 6.6 while the ductile mode contribution as measured from fracture surface striation spacings was characterized by a $B$ value of about 1.5. This exemplifies the second or brittle mode superimposed category of behavior described above. Although we expect a fail-safe behavior at low $\Delta K$ levels for these cobalt-base alloys, there are no data available at present to verify this contention.

A host of nickel-base superalloys, all expibiting planar slip and crystallographic cracking, were found to follow $\Delta \mathrm{K}^{4}(1)$ dependence. In one of these alloys, a high $\gamma^{\prime}$ volume fraction monocrystalline alloy, the attempt was made to $f$ ind a fatigue crack growth rate drop-off at low $\Delta K^{\prime}$ s--none were found, $(3)$ see Fig. 4. Accordingly, it does appear, at least based on the alloys studied, that $\gamma^{\prime}$ strengthened superalloys belong indeed in that third category.

No superalloys thus far have been found to exhibit that nice, ductile, first category behavior. Likely candidates for this behavior may be the lower temperature and more ductile sheet or bar variety superalloys, including the iron containing superalloys.

Clearly, many more fatigue crack growth studies on superalloys are necessary before a healthy situation can exist with respect to truly understanding superalloy crack growth behavior. Further, as reflected by the agglomerated Eq. (25), da/dN resistance, in the case of the realistic smaller cracks, depends on cyclic(26) as well as such non-uniaxial mechanical properties as the plane strain properties. The generation of mechanical property data under more complex states of stress is also necessary for the ranking of creep crack growth resistance in superalloys. We see this determination of plain strain as well as cyclic mechanical properties of superalloys as an urgent task. Despite this lack of basic and relevant information, we will try our best to broadly discuss superalloy crack growth resistance from alloy design viewpoints.

According to the foregoing theory [embodied in Eq. (25)], a higher da/dN resistance would demand increased elastic modulus, cyclic yleld strength, cyclic work hardening exponent, plain strain ductility, and in the case of the planar slip superalloys, the surface energy $\gamma_{s}$. The $\gamma_{s}$ quantity is in many ways an invariant quantity. For a given superalloy base composition, the elastic modulus also remains fairly invariant to small chemistry changes and to microstructural variations. However, it is known that the elastic modulus 
can be manipulated between factors of 1.5 or so through texturing--either solidification or thermomechanically induced. The work hardening behavior is not well understood in superalloys. However, it is known that the work hardening exponent $n$ of planar-slip nickel-base superalloys is low, on the order of 0 , and is reasonably invariable because of the nature of the slip. It is conceivable that $n$ can be higher for the less well-endowed superalloys and for those alloys that are strengthened by carbides or contain oxide dispersoids. At any rate, $n$ is not expected to vary beyond the range of $0-0.2 .(14,26)$ Accordingly, again we are left with strength and ductility to manipulate--and to trade-off.

Achieving high strength in superalloys either through solid solutions, carbides, $\gamma^{\prime}$ or $\gamma^{\prime}$ type coherent precipitates, or oxide dispersoids is at least a task that has been accomplished successfully many times. $(14,27)$ Recently, it has been found, again based on the study of only one superalloy, that monotonic strength of superalloys, i,e, those commonly reported, may be similar to their cyclic yield strengths. $(3)^{\prime}$ 'This correspondence of monotonic and cyclic properties is not always the case, since cyclic (shake-down) strengthening and weakening are observed in, say, steels and aluminum alloys. (26) Although strength can be achieved, achieving it without a substantial loss of ductility $\varepsilon_{F}$ is one of the major problems in alloy design. By the way, our theory, $\Delta K_{n} *$ in $E q$. (25), does indicate that, when applicable, the fail-safe stress intensity can also be raised through increased macroscopic and local $\varepsilon_{F}{ }^{\prime} s$.

A quick survey of superalloy data banks, say Ref. (28), will convince even the skeptic that the perennial conflict between strength and ductility exists also in the case of superalloys. It is well known that ductility drops sharply when precipitates or dispersoids are added to strengthen alloy systems and that this ductility loss is expected to be even more severe when one considers the more realistic plane strain ductility. (14)

The reader also needs no reminder that catastrophic ductility or toughness losses can occur as a consequence of phase instabilities resulting in the tcp and sigma phases, and at times, in the acicular carbides. Superimposed fracture modes in alloys are indeed manifestations of localized weaknesses encountered by a running crack. In cobalt-base alloys these could be grain boundaries either too well endowed with carbides or denuded of carbides. The same applies to nickel-base alloys in which carbides are there to prevent grain boundary sliding. Casting inhonogeneities, unintentional nonmetallic inclusions, and shrinkage pores and voids can also greatly reduce local. $\varepsilon_{F}$ or. toughness. Unintentional trace or tramp elements like tin, lead or arsenic, even in ppm amounts, can cause the superalloys version of temper embrittlement.

On the positive side, it appears that these are at least activities seeking ductility enhancement--tensile as well as stress rupture. The Hf and BC (high boron, low carbon) concepts, $(29)$ directional solidification and monocrystals are certainly developments in the right direction. Powder superalloy technology may also pay dividends with respect to both fatigue and creep crack growth resislances. As mentioned, a runing crack samples local environments. A homogeneous structure, which powder metallurgy can provide, could potentially offer tougher local microstructures to the sampling crack.

With respect to flaw growth resistance of superalloys under creep loading conditions, the amount of experimental data available is again limited but apparently in agreement with the theory that we presented earlier in this paper. As in the case of fatigue crack propagation resistance, it goes without saying that more detalled experimental studies on creep crack growth in superalloys are necessary.

It can be seen from Eq. (24) that creep crack growth resistance can be enhanced by increasing creep strength through alloy design for increased $0 *$, and 
$m$ as well as by increasing the stress rupture ductility through $B$. Creep resistance in superalloys and the manipulations to enhance creep resistance are discussed in some detail elsewhere, e.g., Refs. $(25,30,31)$. Enhancing stress rupture ductility, the general aspects of which we have already discussed, on the other hand, may mean a trade-off with respect to creep resistance. However, again such concepts as BC may be the solution to this problem. Also, the lack of long-time phase stability of the well-endowed superalloys is still a major problem, especially with respect to higher temperature and prolonged applications. One obvious solution is to make the alloys with fewer components, enough in variety to promote corrosion resistance, and depend on oxide particles for strengthening. This indeed is the attractiveness of oxide dispersion strengthened binaries and ternaries. However, another thrust: should be in the analytical direction of predicting phase stabilities through continuations of, say, the PHACOMP fype studies $(32,33)$ and the more recent pair-potential model studies. $(34-37)$

As a concluding point, we would like to remind the reader of the fact that synergistic creep-fatigue interactions and the attendant effects on flaw growth behavior of superalloys have not been touched upon in this paper. Certainly, our own analytical efforts will be in the direction of either incorporating thermal activation into fatigue crack growth or including cyclic constraints into creep crack growth, or both.

\section{ACKNOWLEDGMENTS}

We are grateful to the National Aeronautics and Space Administration, and to the National Science Foundation for supporting portions of this work through grants NASA-NSG-3050 and NSF-DMR75-09878, respectively. We thank Mr. Michael Fischer for editing and typing the manuscript. 


\section{REFERENCES}

1. C. A. Rau and L. H. Burck, Eng. Frac. Mech. 2, 211 (1971); also C. A. Rau, Private communication, Failure Analys is Inc., Palo Alto, CA (1975).

2. T. Ohmura, R. M. Pelloux and N. J. Grant, Eng. Frac. Mech. 5, 909 (1973).

3. S. Purushothaman, D. Eng. Sc. thesis, Columbia University, New York, NY (1976).

4. J. M. Barsom, ASTM-STP 486, 1 (1971).

5. R. C. Bates and W. G. Clark, Trans. Q. ASM $\underline{62}, 380$ (1969).

6. R. 0. Ritchie and J. F. Knolt, Acta. Met. 21, 639 (1973).

7. S. Floreen, Met. Trans. 6A, 1741 (1975).

8. M. J. Siverns and A. T. Price, Int. J. Frac. 9, 199 (1973).

9. G. J. Neate and M. J. Siverns, Proc. Int. Conf. on Creep and Fatigue in Elevated Temperature Applications, Publication \#13, P. 243.1, Inst. of Mech. Engrs., Philadelphia, PA (1973).

10. P. C. Paris, Chapter in FATIGUE--AN INTERDISCIPLINARY APPROACH, Syracuse University Press, Syracuse, NY (1964).

11. P. C. Paris and G. C. Sih, ASTM-STP 381,30 (1966).

12. J. R. Rice, ASTM-STP 415, 247 (1967).

13. G. T. Hahn, Proc. of the Battelle Colloquium on Fundamental Aspects of Structural Alloy Design, Paper IV-2, Harrison Hot Springs and Vancouver, British Columbia, (Sept. 1975).

14. J. K. Tien, Ibid., Paper III-2.

15. M. Gell, G. R. Leverant and C. M. Wells, ASTM-STP 467, 113 (1970).

16. J. K. Tien and R. P. Gamble, Met. Trans. 2, 1933 (1971).

17. S. Purushothaman and J. K. Tien, Scripta Met. 9, 923 (1975).

18. J. W. Cahn, Acta. Met. 8, 554 (1960).

19. J. W. Cahn, W. B. Hillig and G. W. Sears, Acta. Met. 12, 1421 (1964).

20. W. K. Burton, N. Cabrera and F. C. Frank, Phil. Trans. Roy. Soc. A243, 299 (1950).

21. J. K. Tien, Ph.D. thesis, Carnegie-Mellon University, Pittsburgh, PA (1969).

22. A. G. Evans and L. J. Graham, Acta Met. 23, 1303 (1975).

23. F. C. Monksman and N. J. Grant, Proc. ASTM $\underline{56}, 834$ (1956).

24. K. Aning, D. Eng. Sc, thesis, Columbia University, New York, NY (1976).

25. J. K. Tien, M. Malu and S. Purushothaman, Chapter in STRENGTHENING MECHANISMS AND ALLOY DESIGN, J. K. Tien and G. S. Ansell (eds.), Academic Press, New York, NY, In press, (1976). 
26. "Monotonic and Cyclic Properties of Metals and Alloys," Ford Motor Company, Dearborn, Michigan (1970).

27. R. F. Decker, Proc. Symposium on Steel Strengthening Mechanisms, p. 147 , Climax Molybdenum Company, Greenwich, Connecticut (1970).

28. "High Temperature High Strength Nickel Base Alloys," International Nickel Company, New York, NY (1964).

29. D. H. Maxwell, J. F. Baldwin and J. F. Radavich, "A New Concept in Superalloy Ductility," Presented at the Paris Air Show, Le Bourget, Paris (1975).

30. K. Aning, F. T. Furillo, J. M. Davidson, S. Purushothaman and J. K. Tien, "Creep and Stress Rupture in Nickel Base Superalloys," This conference.

31. M. Malu and J. K. Tien, Acta Met. 22, 145 (1974).

32. L. R. Woodyatt, C. T. Sims and H. J. Beattie, Trans. AIME 236, 519 (1966).

33. W. J. Boesch and J. J. Slaney, Met. Prog. 86, 109 (1964).

34. E. S. Machlin, Acta Met. 22, 95 (1974).

35. E. S. Machlin, Acta Met. 22, 109 (1974).

36. E. S. Machlin, Acta Met. 22, 367 (1974).

37. E. S. Machlin, Acta Met. 22, 1433 (1974).

38. W. Elber, Eng. Frac. Mech. 2, 37 (1970). 